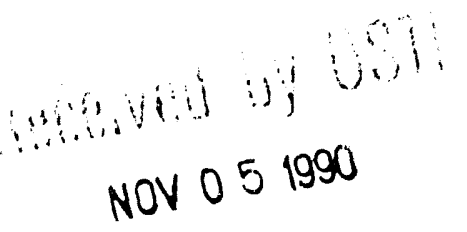

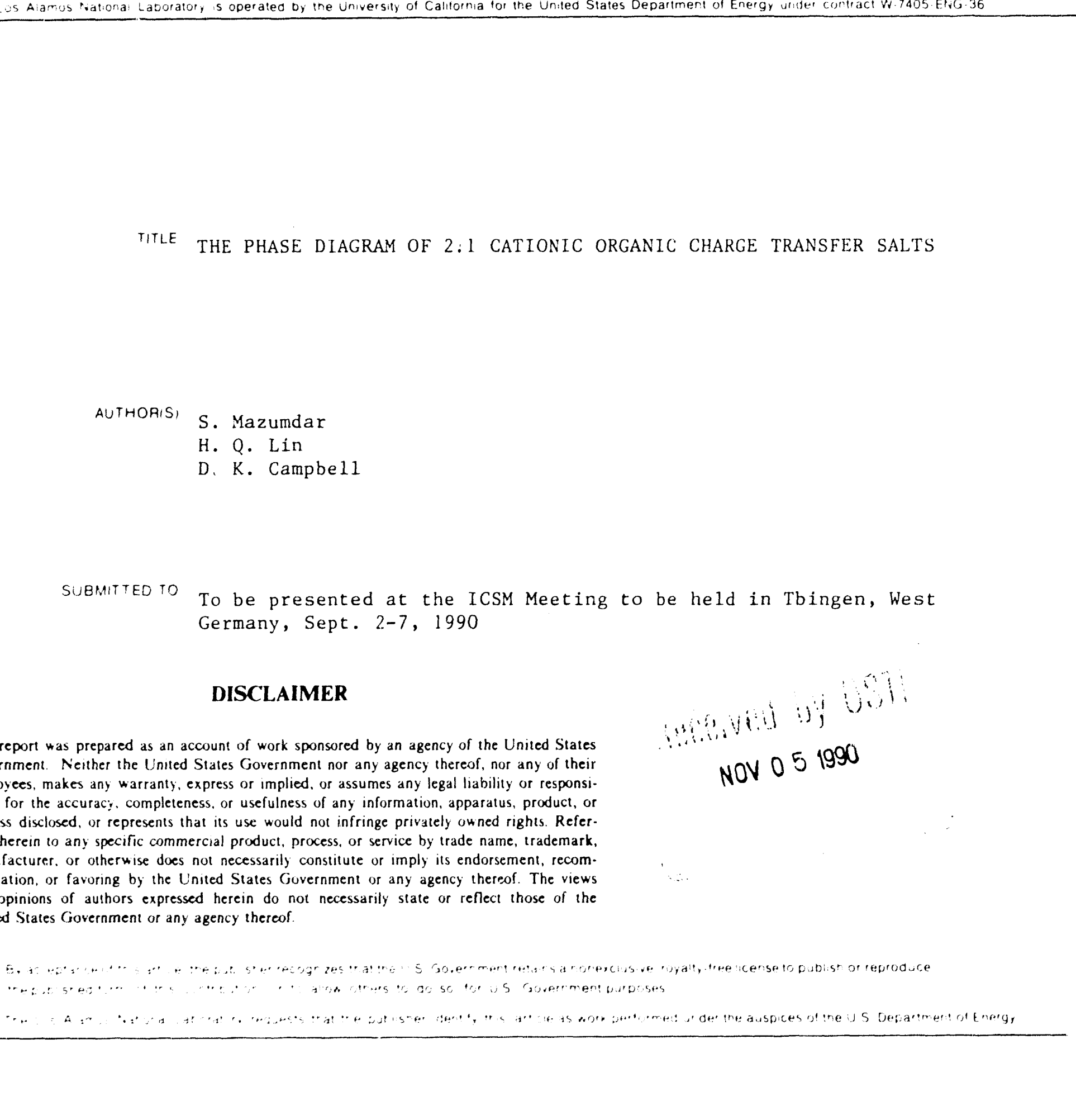

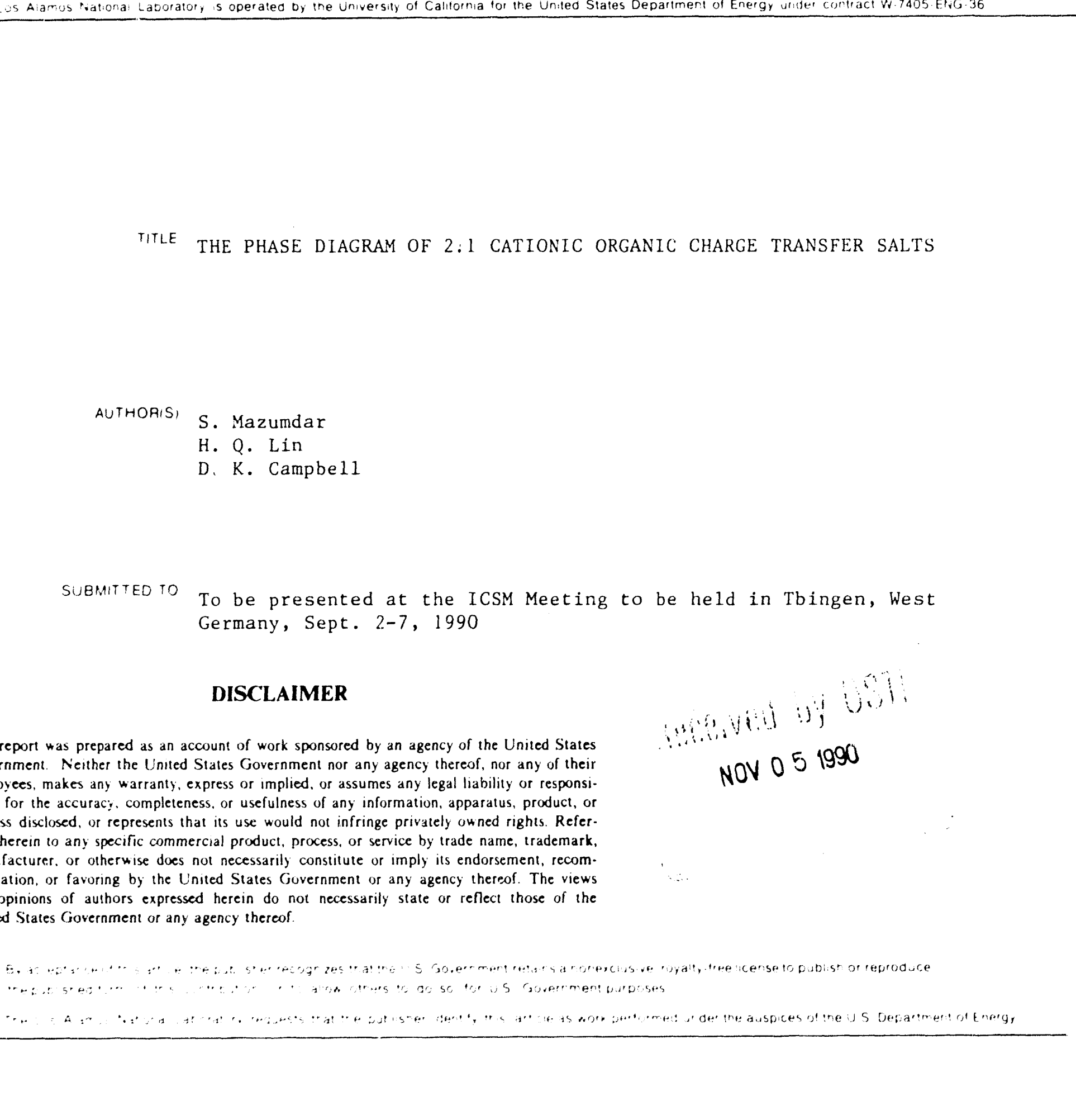

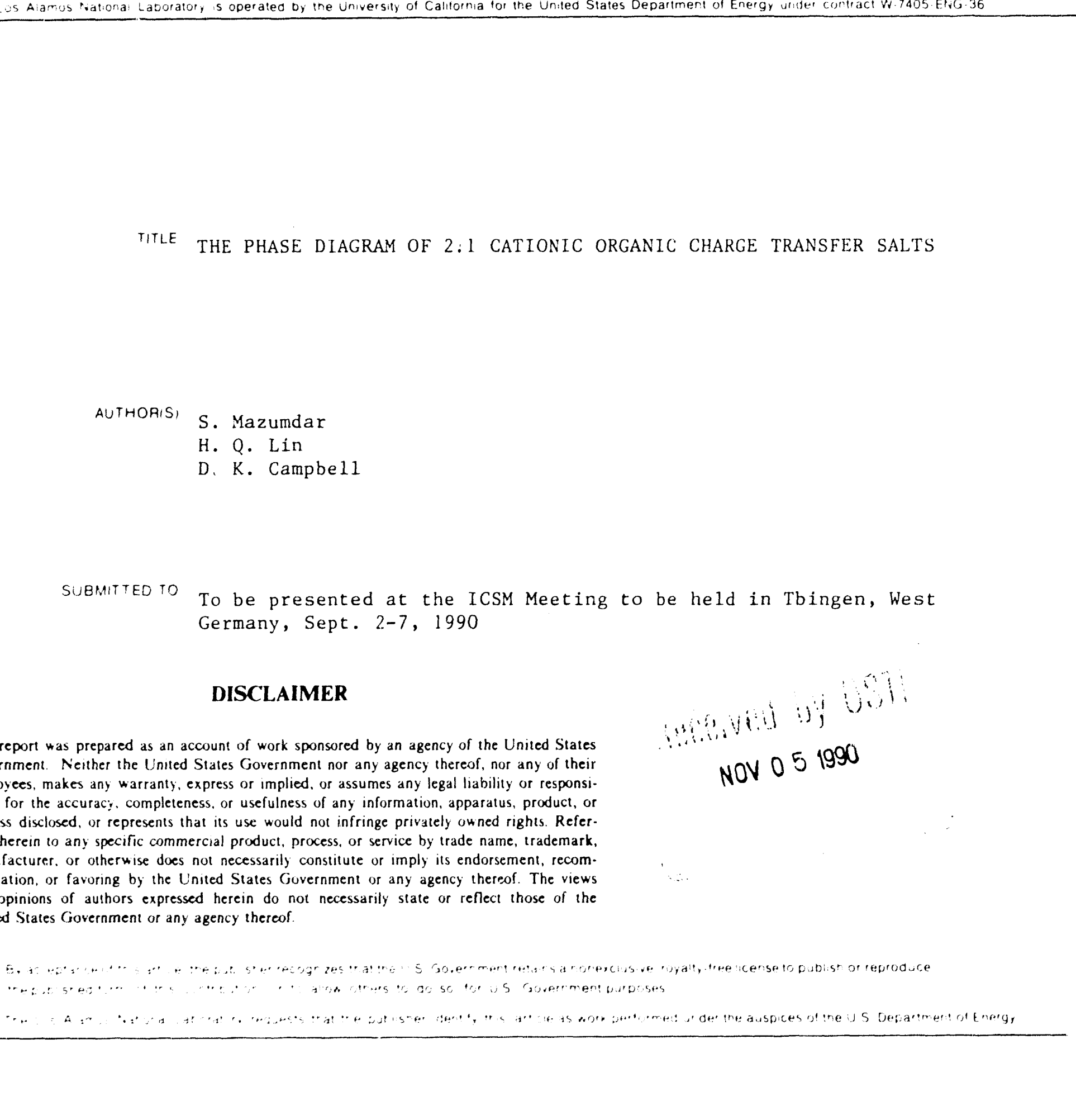

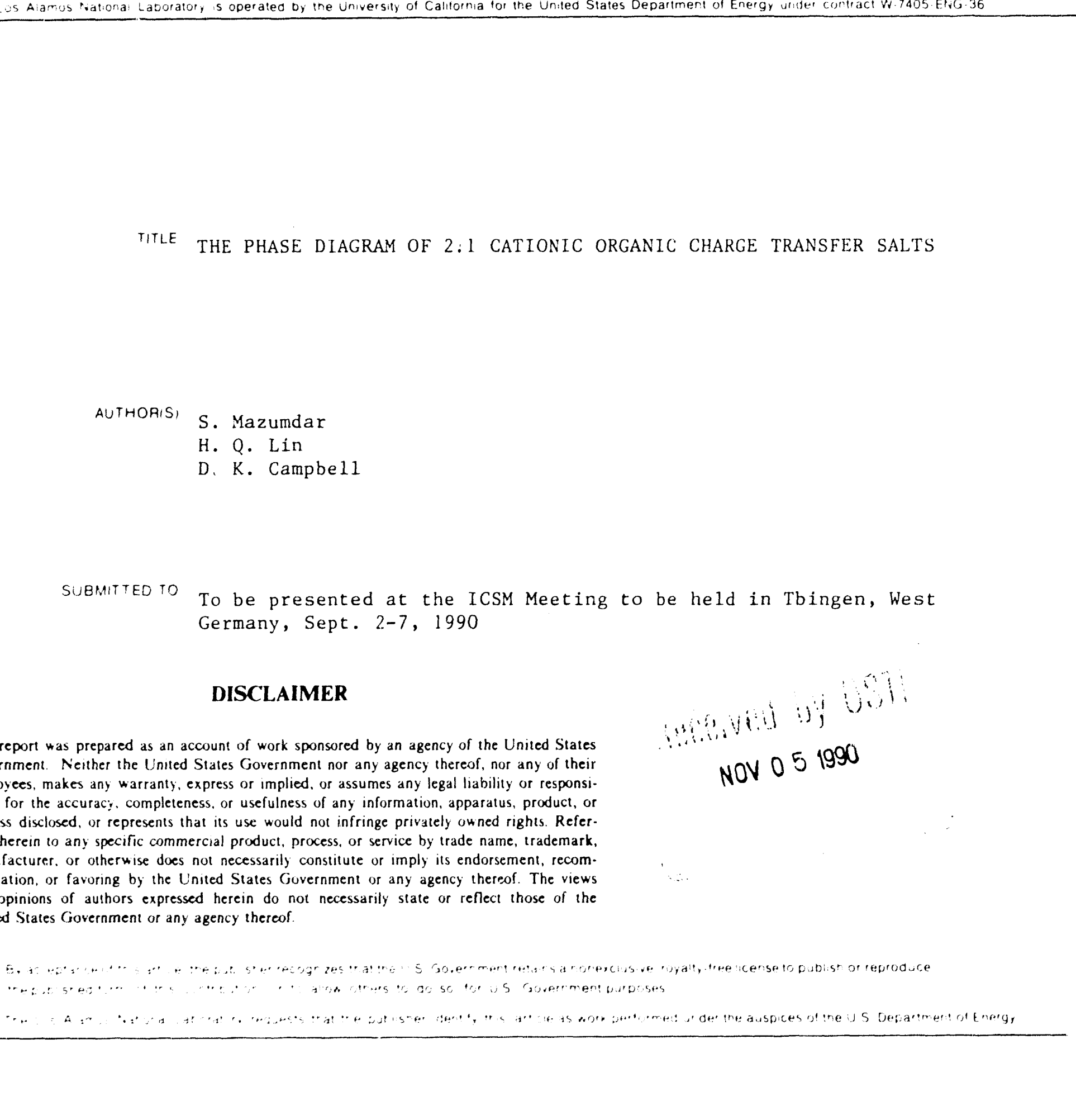

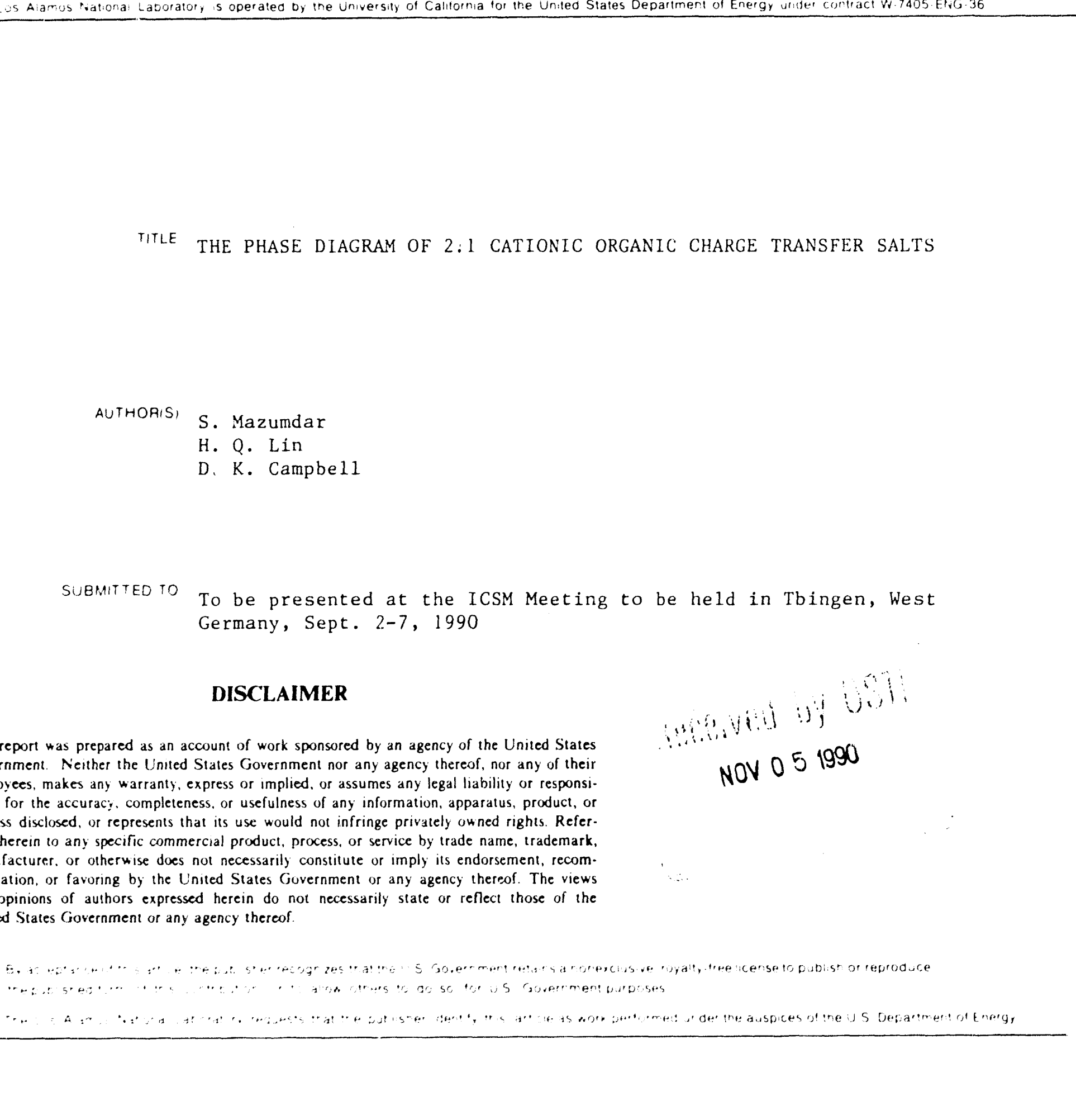

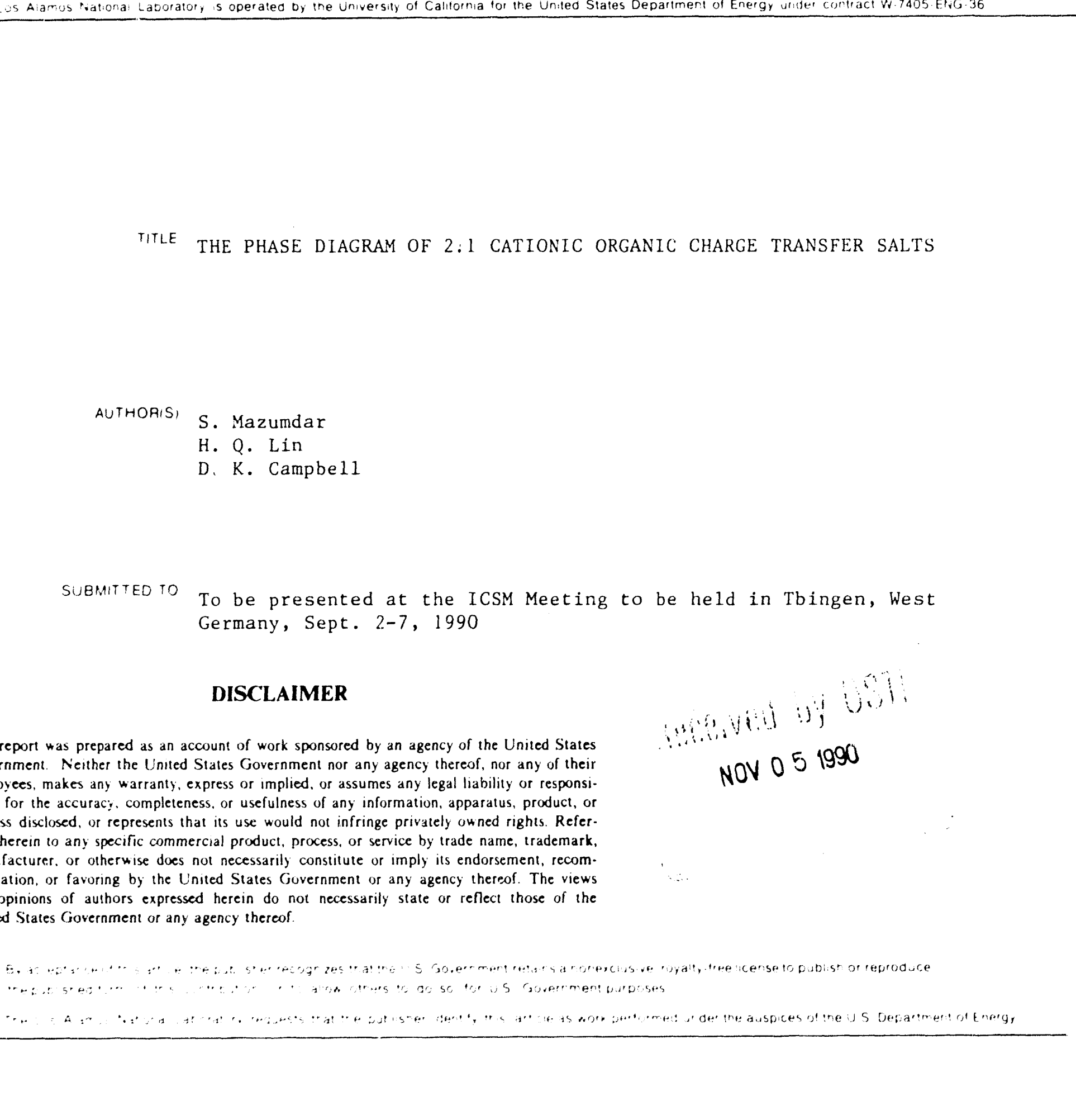

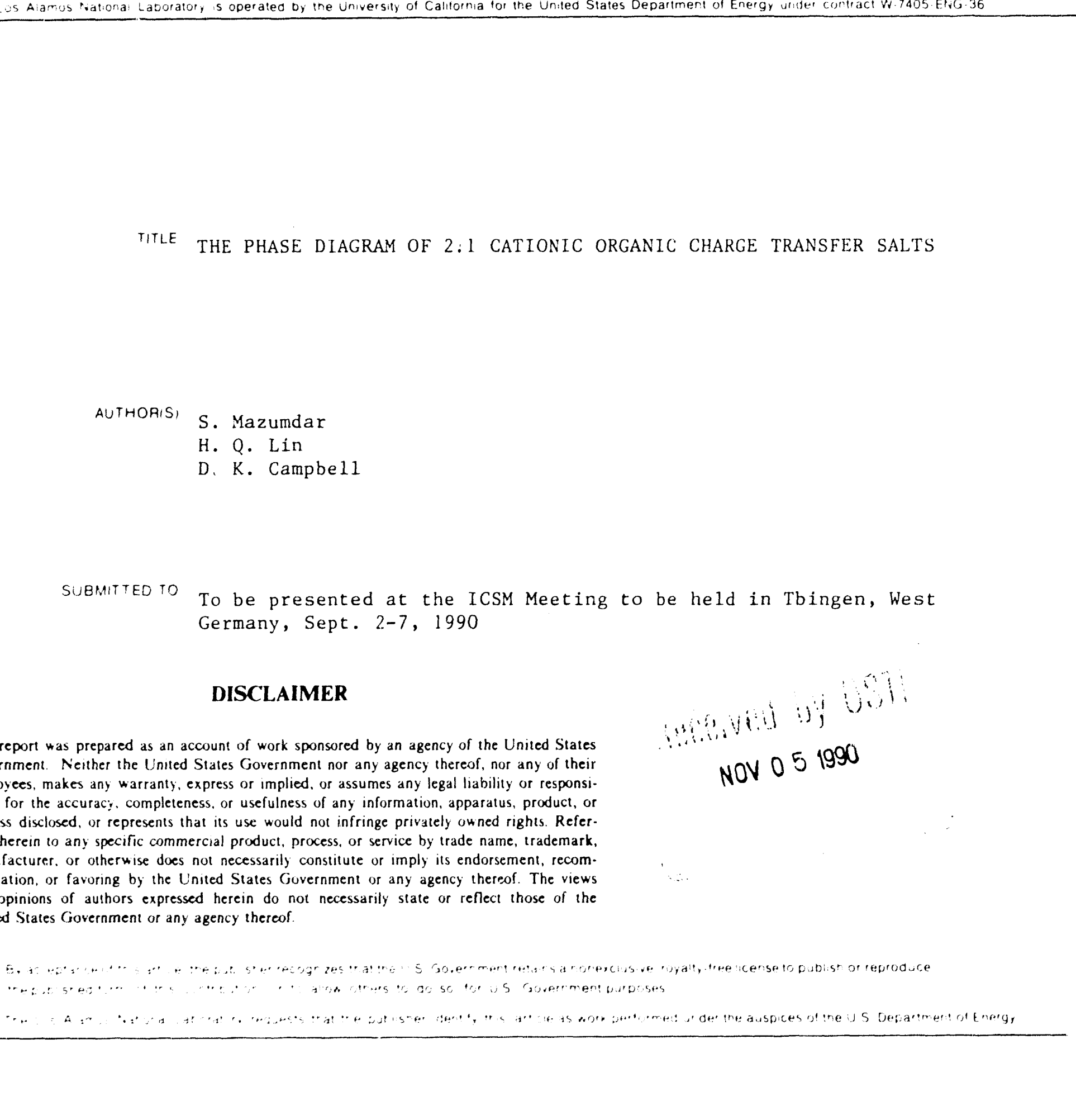

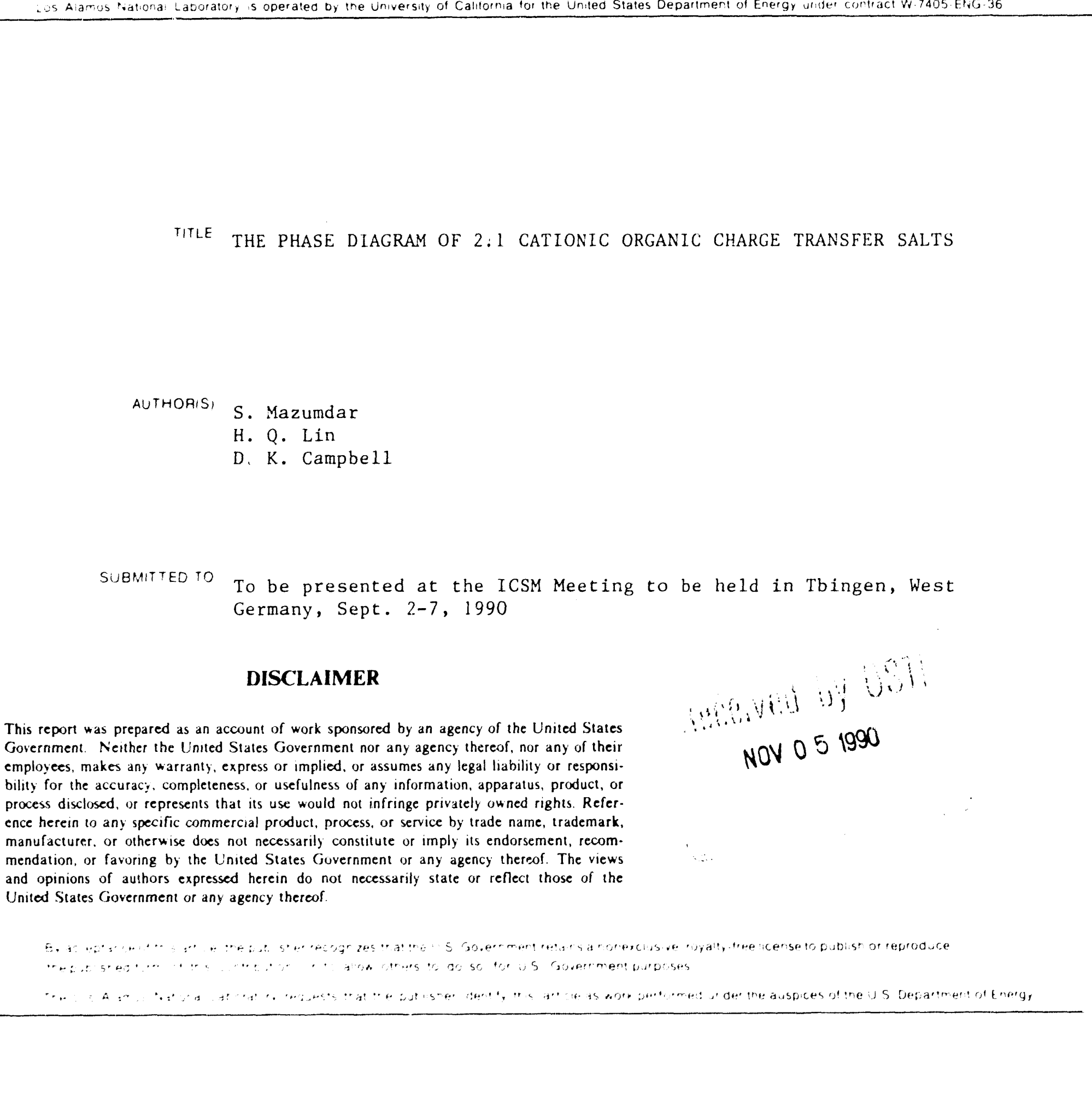

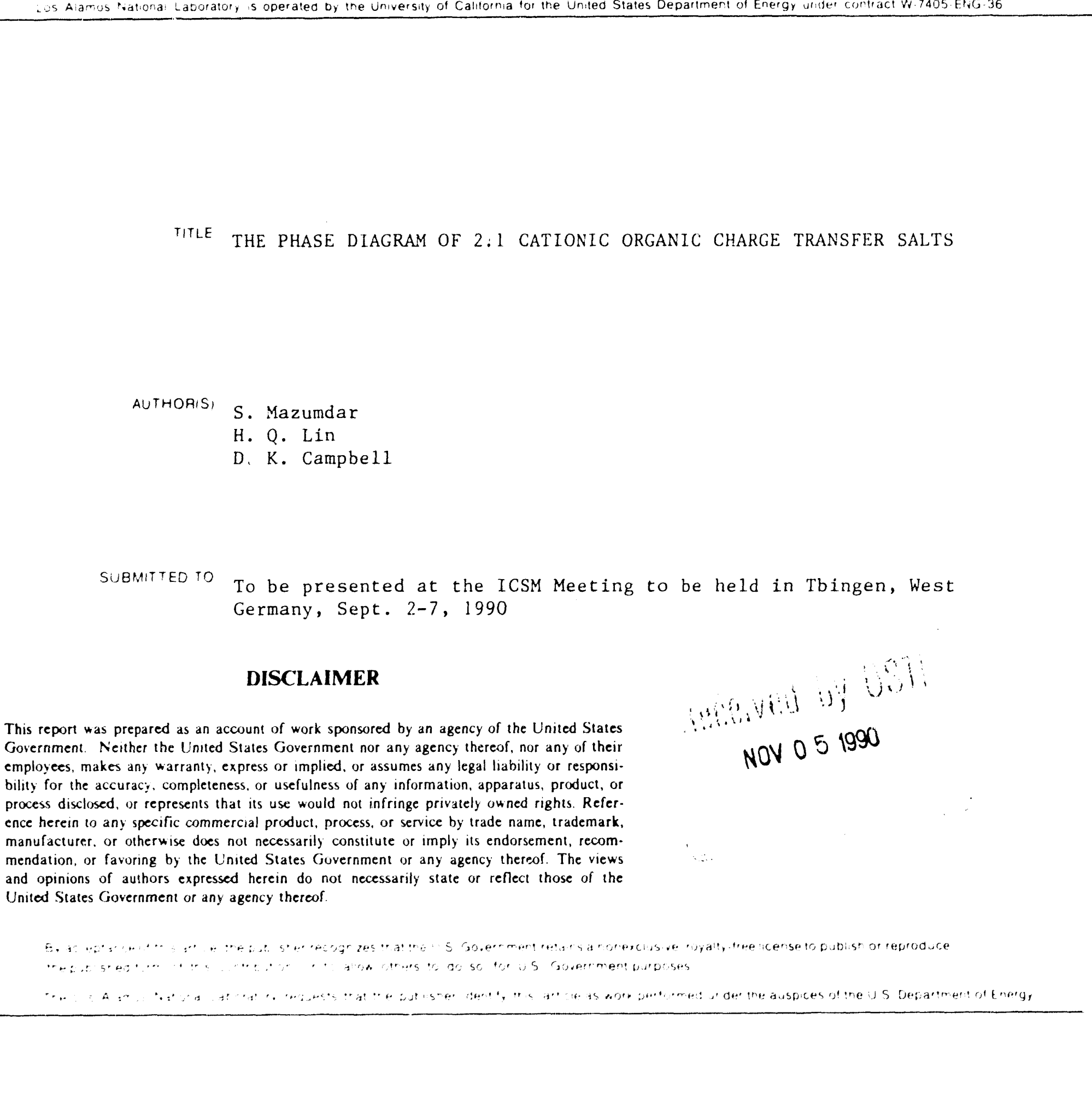

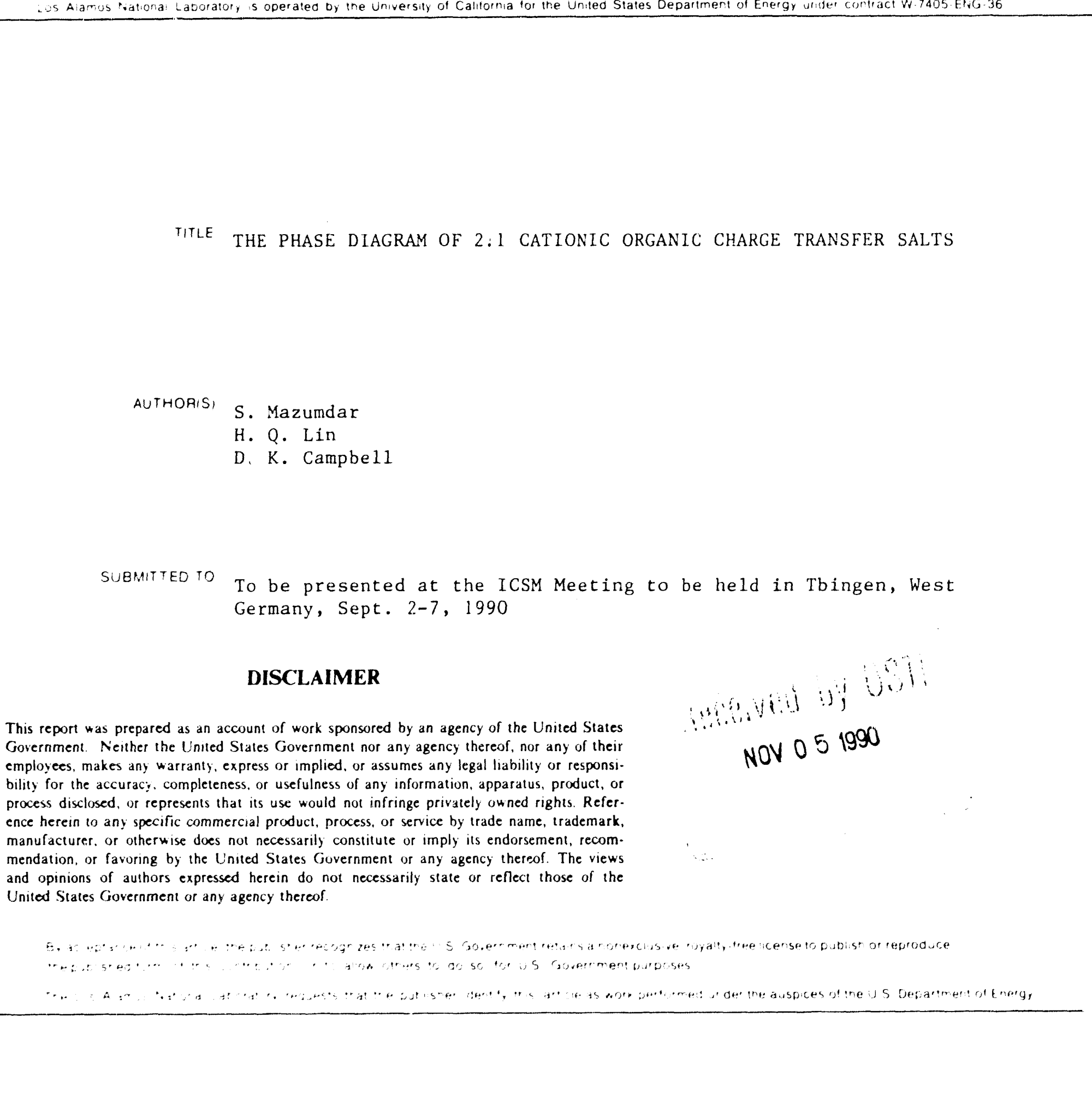

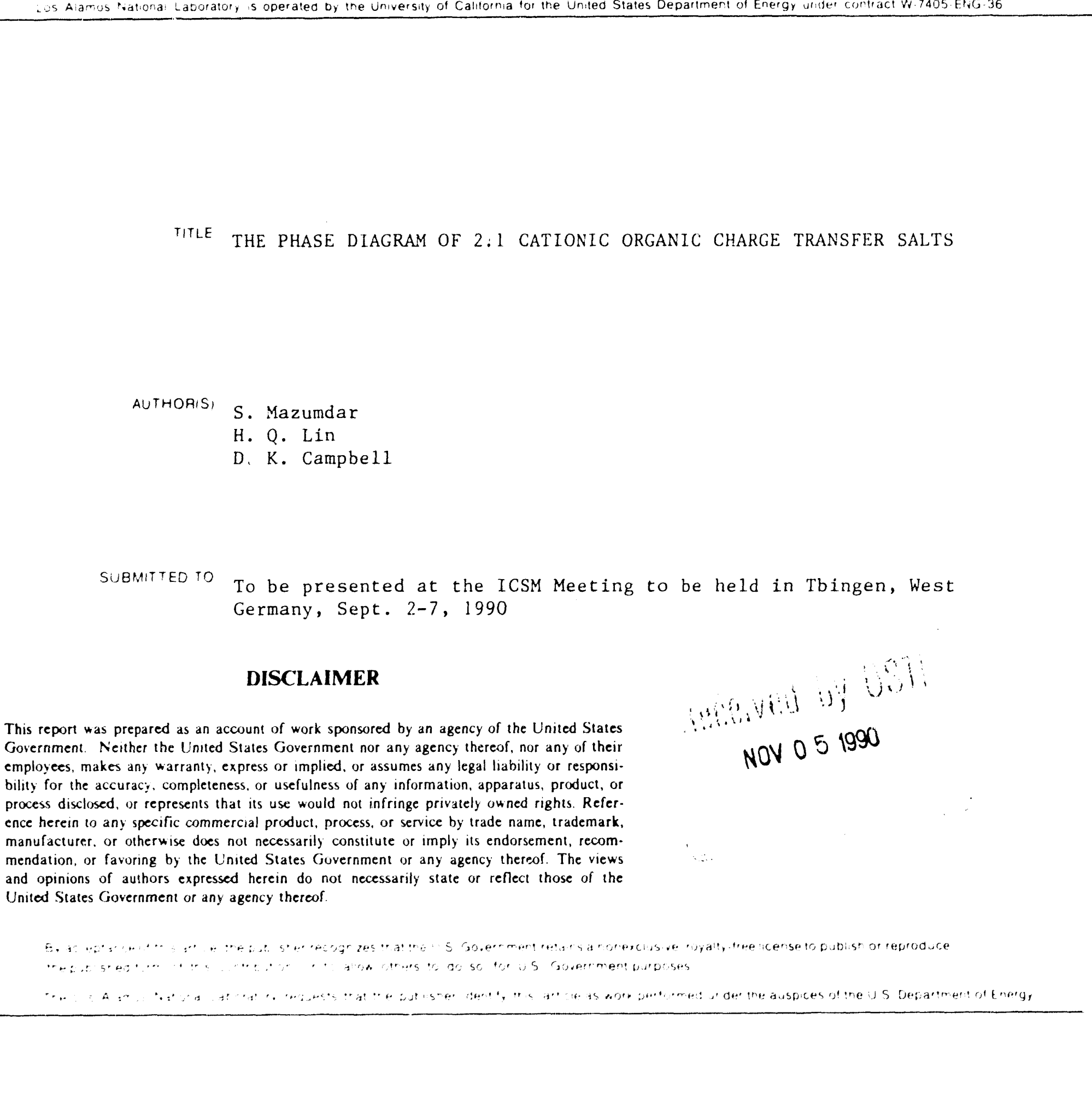

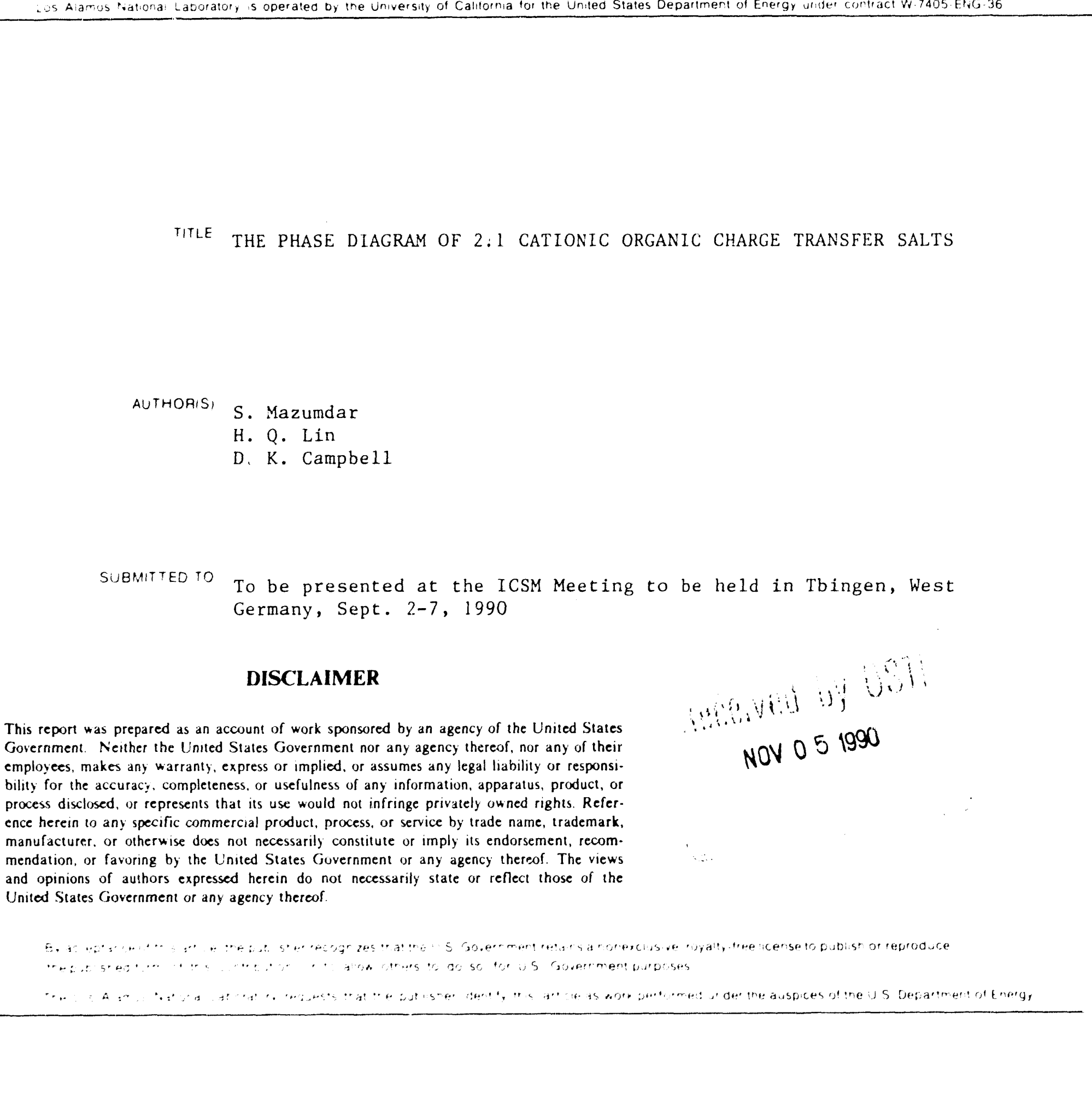

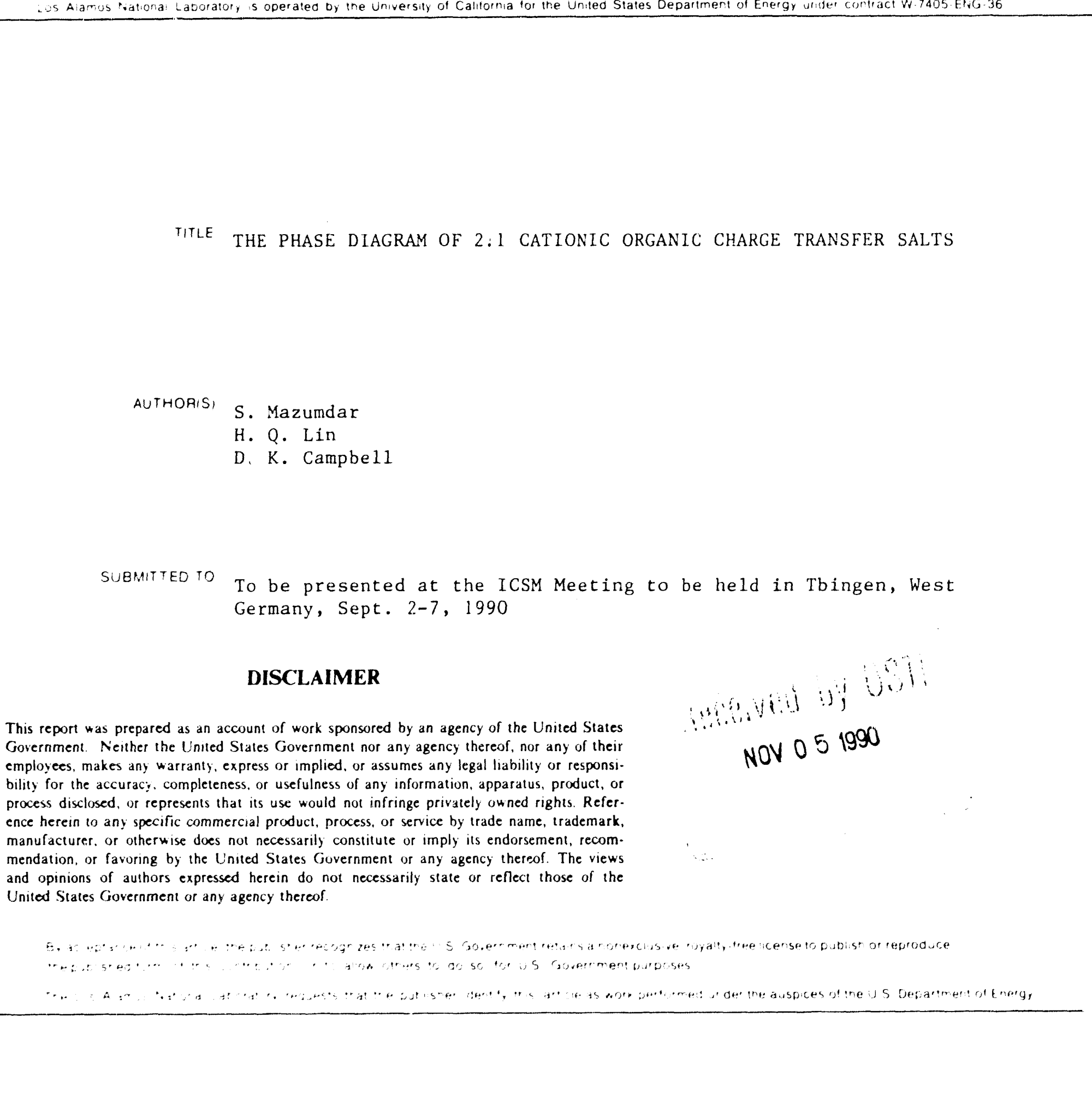

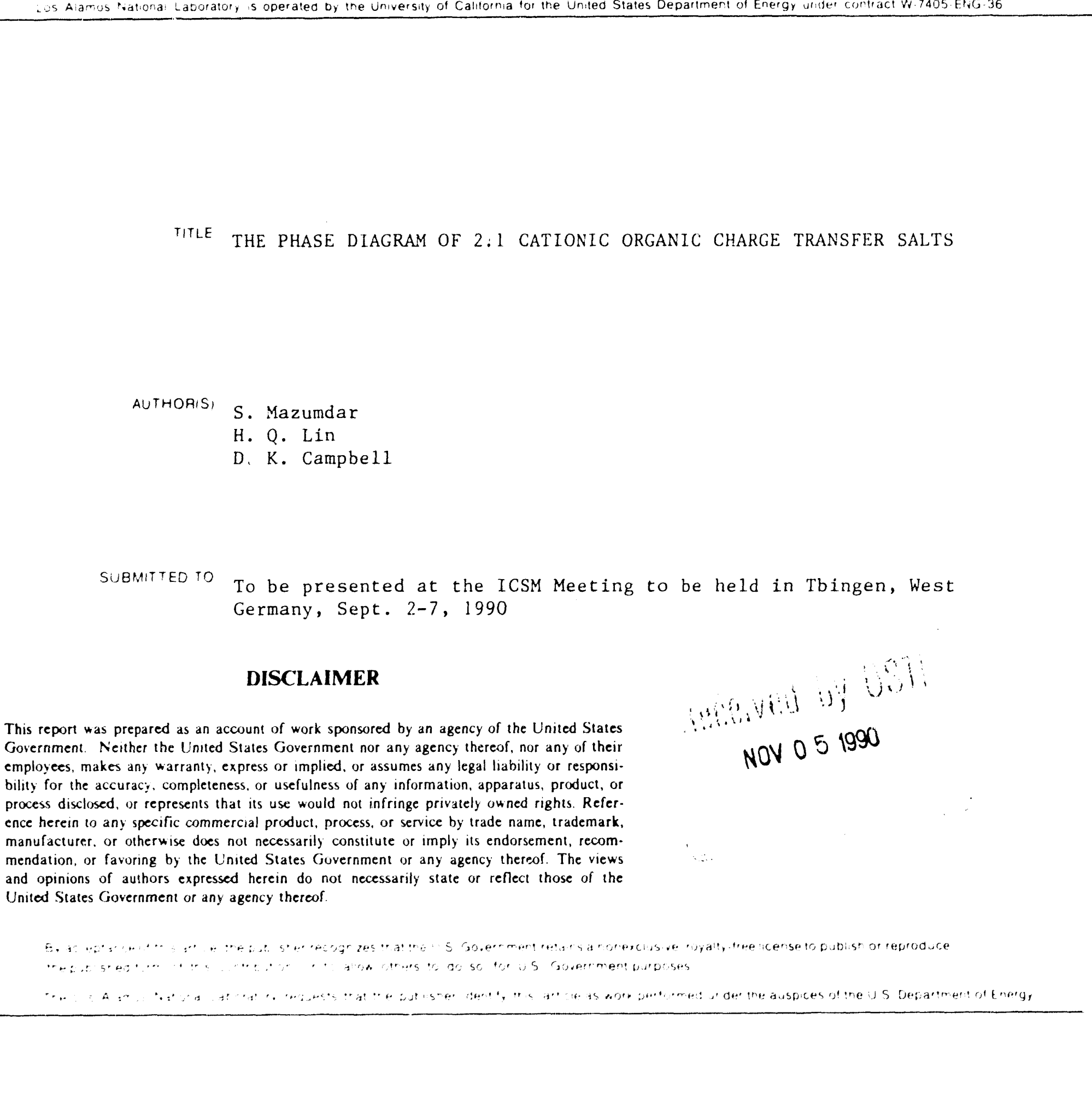

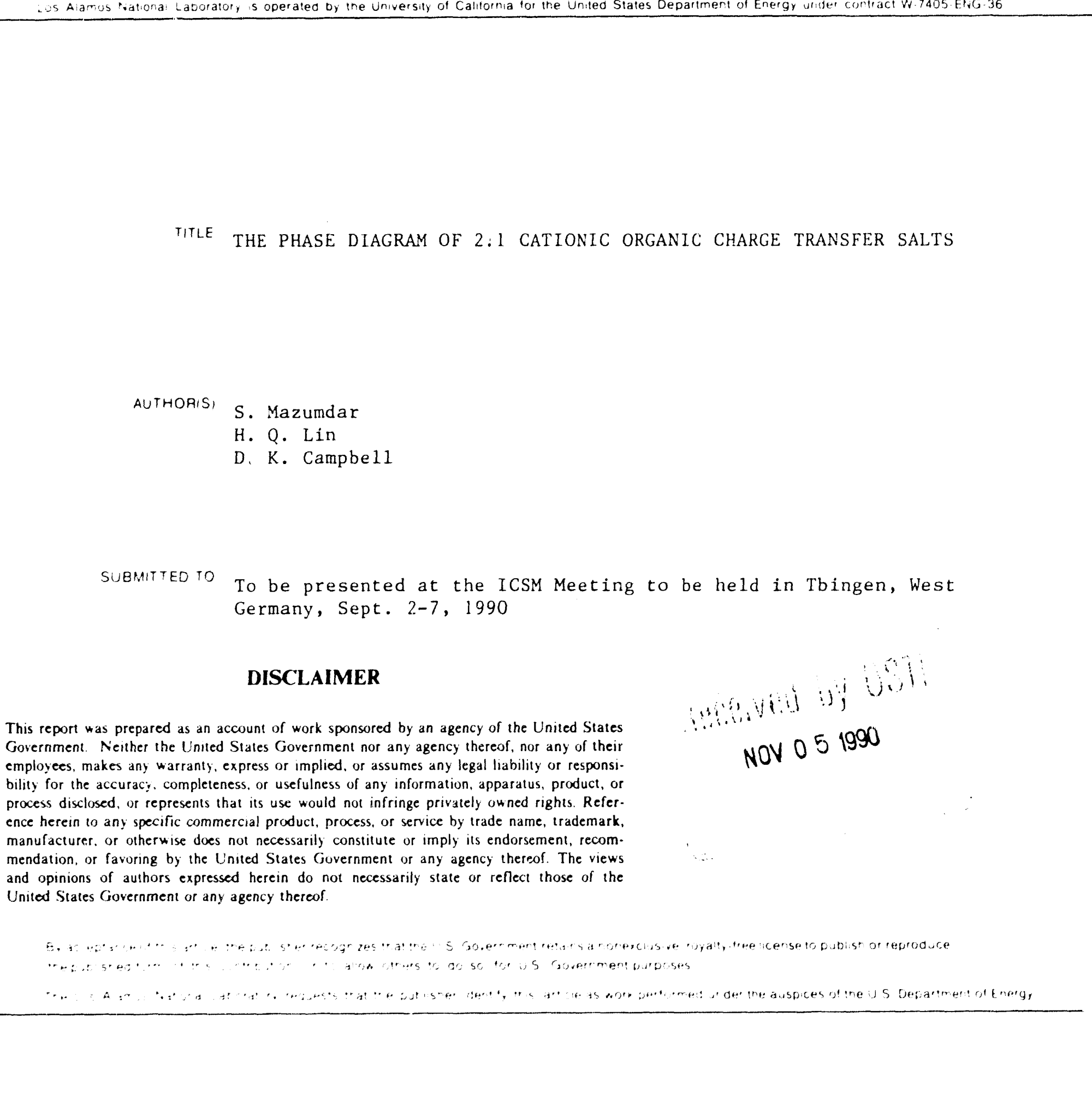

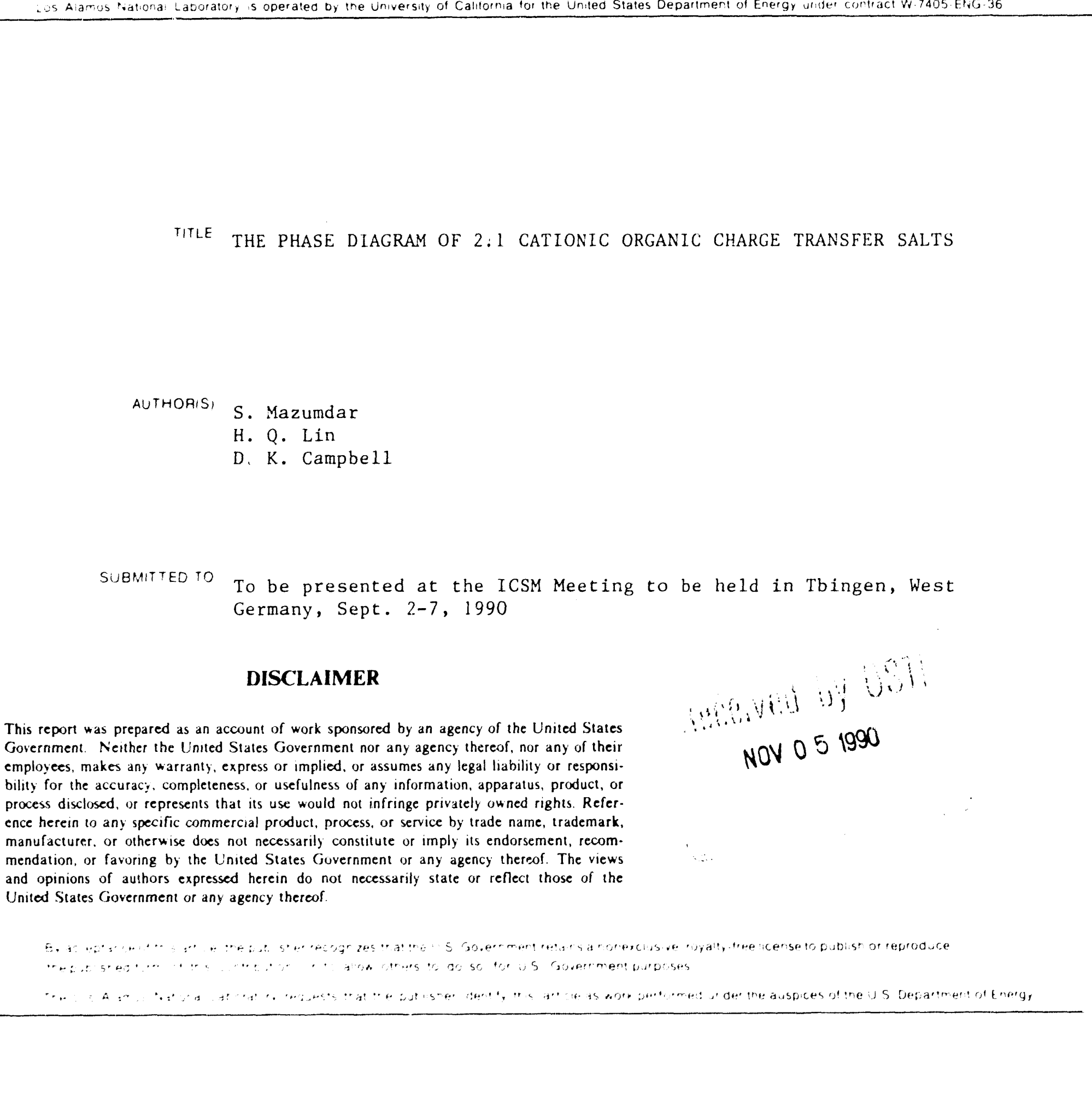

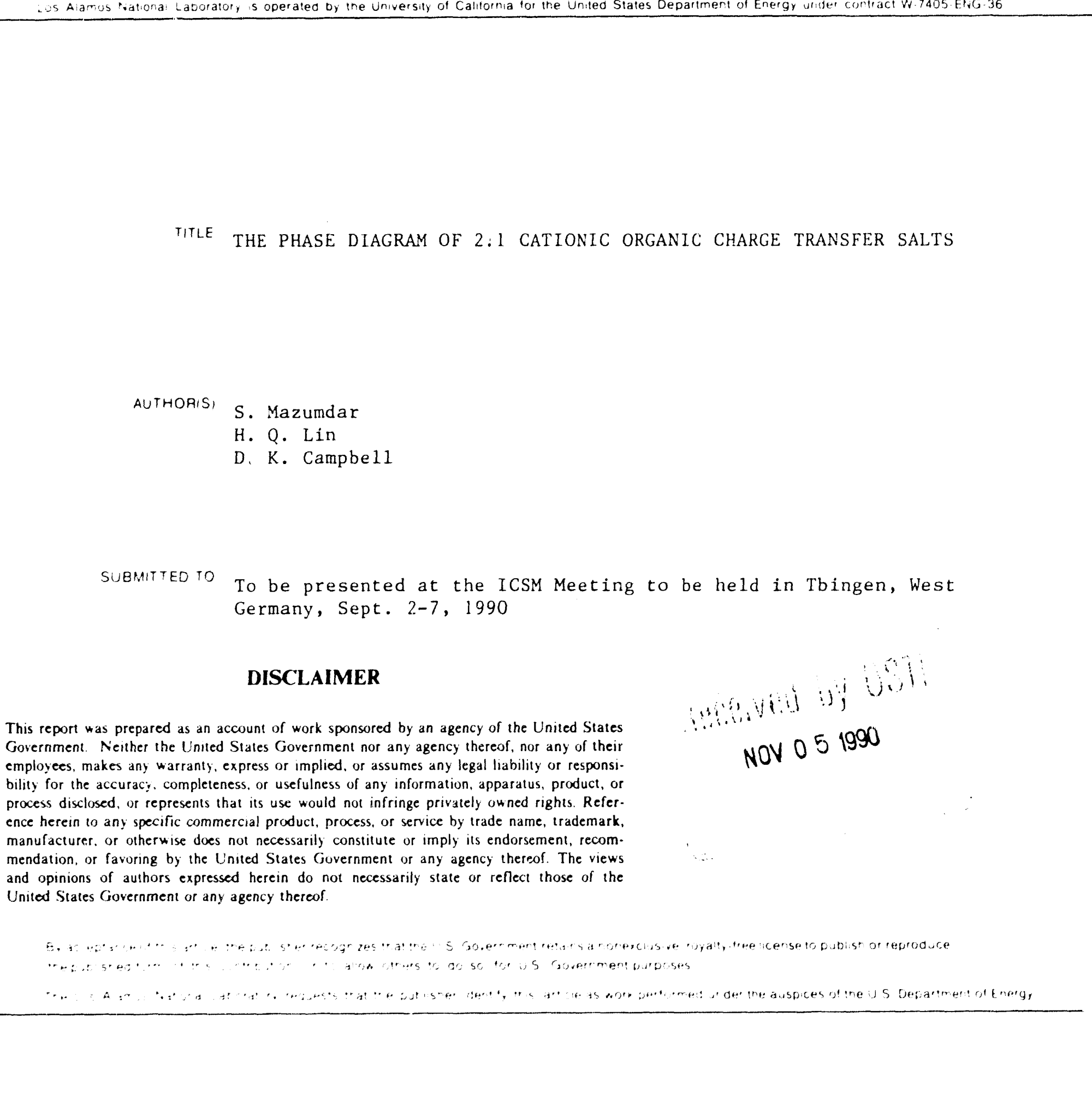

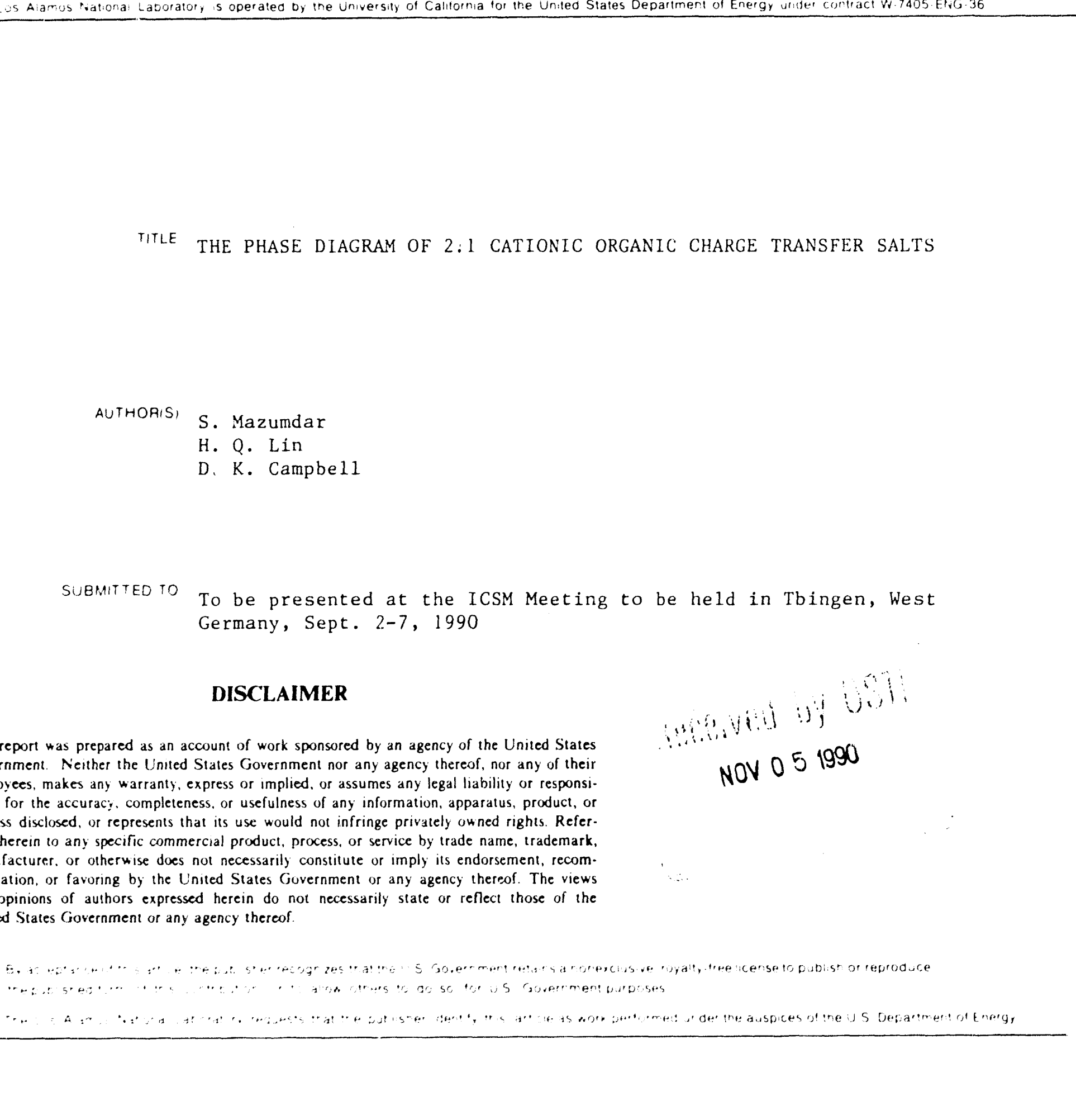

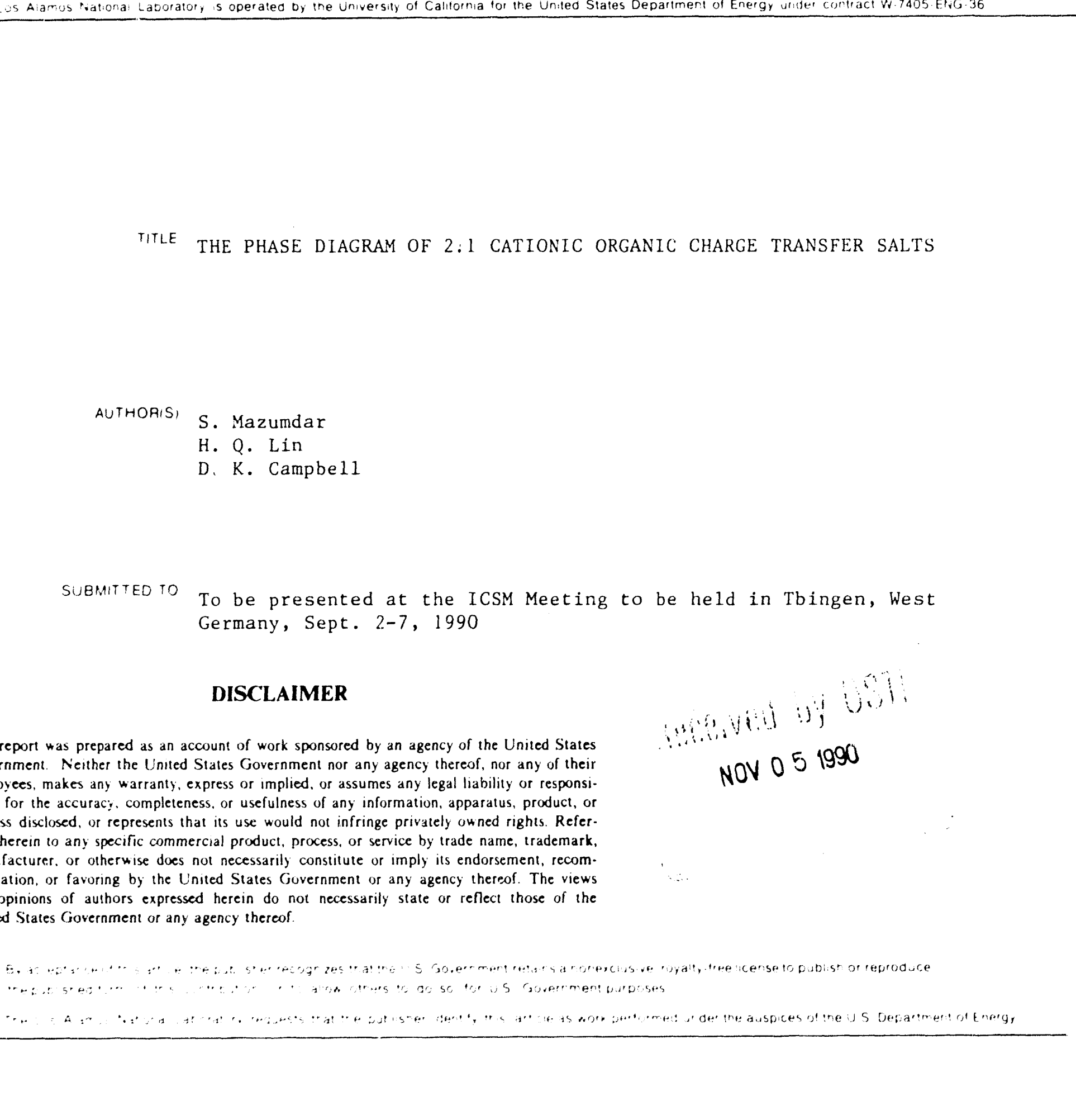

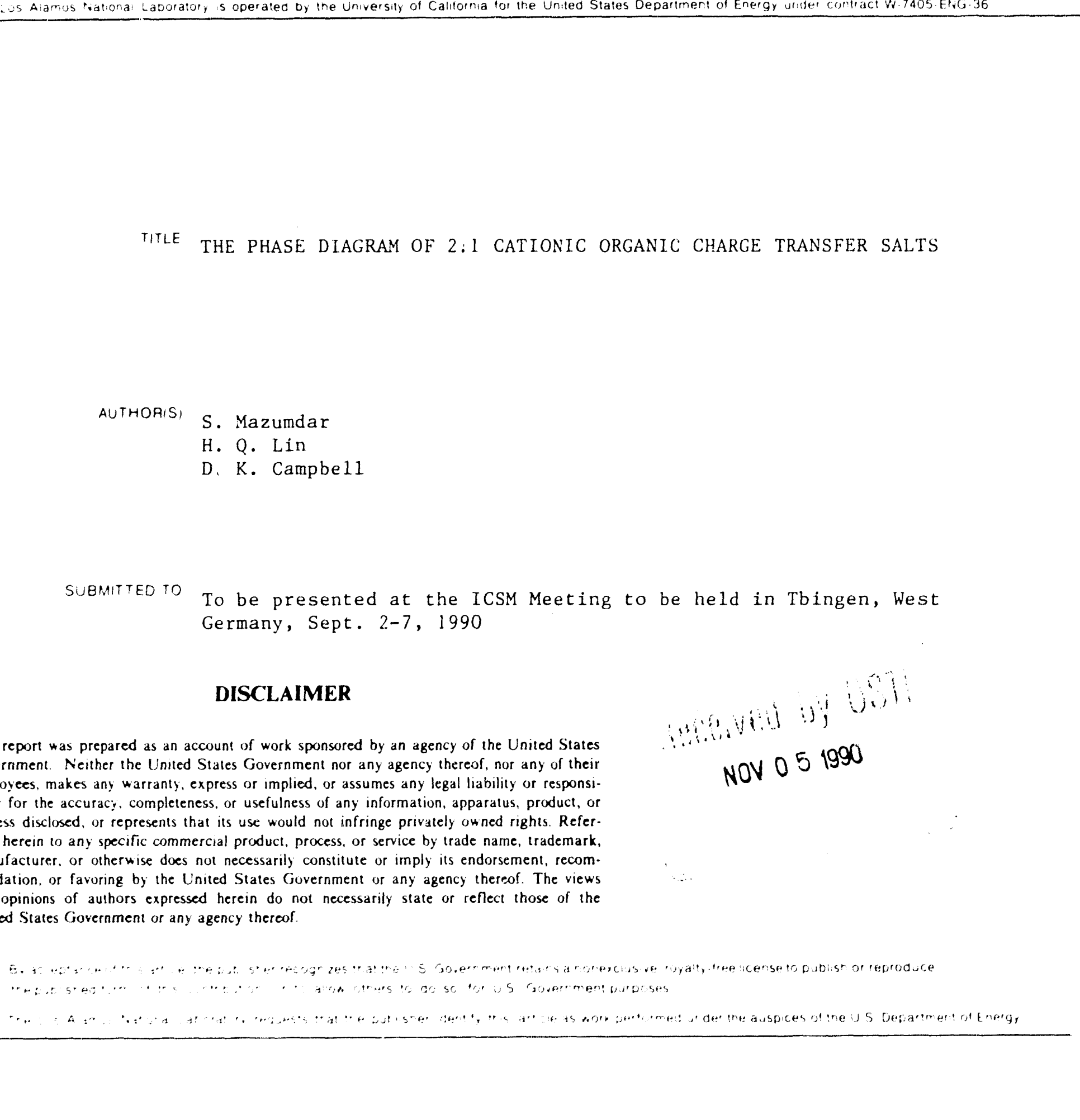

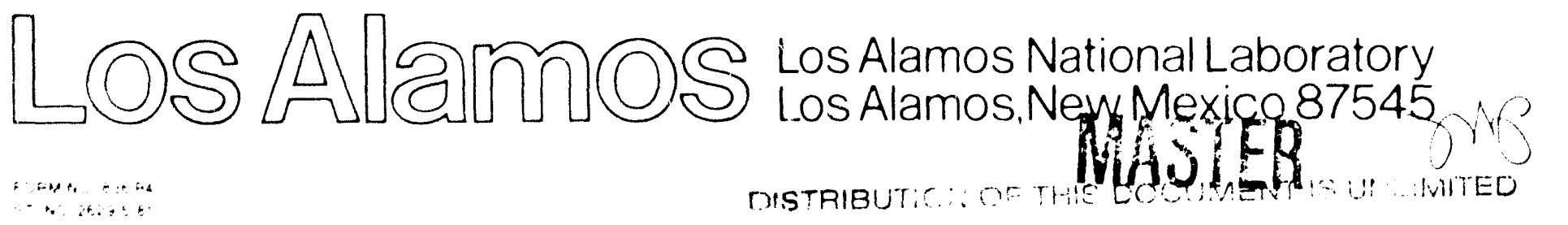




\section{THE PHASE DLAGRAM OF 2:1 CATIONIC ORGANIC CHARGE TRANSFER SALTS}

\section{S. MAZUMDAR}

Dept. of Physics, Univ. of Arizona, Tucson, Ariz. 85721 (USA)

H. Q. LIN and D. K. CAMPBELL

Center for Nonlinear Studies, MS B258, Los Alamos National Laboratory, Los Alamos, NM 87545 (USA)

\section{ABSTRACT}

We propose a new theoretical approach, stressing the role of Coulomb interactions and bandfilling effects, to describe the phase diagram of the 2:1 cationic organic charge transfer salts, including those exhibiting field induced spin density wave (FISDW) behavior. We predict three distinct nonsuperconducting phases.

\section{INTRODUCTION}

When magnetic fields $(\mathrm{H})$ are applied at low temperatures $\left(\sim 5^{\circ} \mathrm{K}\right)$ to the superconducting (TMTSF) ${ }_{2} \mathrm{X}$ salts [1], superconductivity first gives way to a "metallic" state, albeit with possible unusual properties. Then for fields $\mathrm{H}>\mathrm{H}_{f}(\sim 5$ Tesla) a cascace of transitions to semimetallic SDW phases (as deduced from NMR data [2]) is observed. The explanations of this FISDW phenomenon involve the gradual "one dimensionalization" of the anisotropic 2-D Fermi surface due to the magnetic field, and an accompanying sequence of nesting instabilities [3-5]. Within these "standard" theories, the phase at very high fields is expected to be a commensurate, large gap SDW semiconductor. These theories thus fail to account for the recent observation in the perchlorate salt $\left(\mathrm{X}=\mathrm{ClO}_{4}\right)$ of the VFHT $[6,7]$, where for $\mathrm{H}$ $>17$ Tesla it is found the transition temperature $T_{S D W}$ begins to decrease with field, and actually goes to zero for $H \cong 25.30$ Tesla. At present, there exists only one "nonstandard" [8] theoretical approach that explains the VFHT. Importantly, within this approach, the VHF phase is predicted to be metailic; experimentally, however, the material exhibits high resistivity, activated conductivity, vanishing Hall signal and diamagnetism, all characteristics of a semiconductor or a semimetal. Thus it appears that, despite its successes [8], the "nonstandard" approach is incomplete.

In the present article we argue that an anisotropic, quasi-2-D extended Irubbard Hamiltonian, in which both nearest-neighbor Coulomb interactions and the quarter-filled nature of the organic stacks are incorporated [9], not only explains the VHFT but also unifies the theoretical understariding of the pressure and magnetic field dependence of the phases of many other familien of structurally similar 2:1 cationic organic charge transfer salts, including the TMTTF, BEDT-TTF, DM and MDT-TTF 
materials. We show that the model predicts three different nonsuperconducting phases, depending on the extent of the anisotropy. Further, pressure, magnetic field or any other effects that change the effective anisotropy can drive transitions among the phases. The FISDW behavior can be included as in the original theories, and it has been suggested [10] that the same model can provide a mechanism for the superconductivity seen in some of these systems.

\section{THEORETICAL MODEL AND NUMERICAL RESULTS}

We consider the sing.orband, anisotropic, quasi-2D extended Hubbard model,

$$
H=-\sum_{i, \sigma}\left(t_{x} c_{i, \sigma}^{\dagger} c_{i+x, \sigma}+t_{y} c_{i, \sigma}^{\dagger} c_{i+y, \sigma}+h . c .\right)+U \sum_{i} n_{i \uparrow} n_{i \downarrow}+\sum_{i}\left(V_{x} n_{i} n_{i+x}+V_{y} n_{i} n_{i+y}\right),
$$

on a rectangular lattice, but realistic lattice structures can be incorporated later. The operators and parameters in (1) have their standard interpretations; the $x$-axis is chosen as the stacking axis. The implicit parameter $\rho$, the number of holes per site on the organic stack, is very significant, and is fixed at 0.5 (quarter-filling). Numerical calculations are then performed for various anisot sopies $\left(0<t_{y} / t_{x}<\right.$ $\left.1,0<V_{y} / V_{x}<1\right)$ to determine the dominant broken symmetry.

Before presenting our numerical results, we indicate our physical arguments suggesting the possibility of three distinct phases for the ground state of the Hamiltonian (1) for $\rho=0.5$. In the 1-D limit $t_{y}=0=V_{y}$, it is known that the SDW, which is a continuous broken symmetry, can never occur as a distinct phase with long-range order. Indeed, the dominant broken symmetry, for small enough $V_{x}$ (relative to $U$ ) is the bond onder wave (BOW) in which the order parameter is the bond order (charge transfer); given any coupling at all to the lattice, this leads to either a Peierls (weak coupling) or a spin-Peierls (SP) (strong coupling) phase. $[9,11]$ In the isotropic case $\left(V_{y}=V_{x}, t_{y}=t_{x}\right)$, it is known for $\rho=1$ that the BOW does not occur [10], and that the SDW dominates (as it can in a 2-D ground state). Intuitively, we expect the relative suppression of the BOW to occur even for $t_{y}$ considerably less than $t_{x}$, and for $\rho=0.5$ as well as for $\rho=1$. However, while for $\rho=1$ the SDW becomes progressively stronger with increasing $t_{y}$, for $\rho=0.5$ (and $V \neq 0$ ), a spin frustration can occur, destroying the SDW. This is sketched in Fig. 1, which for simplicity depicts the large $U$ limit. These considerations lead us to expect three distinct possible ground states for the Hamiltonian in Eq. (1): (i) a SP (BOW) phase, for $t_{y}<t_{y_{1}}$; (ii) a SDW phase (including FIDSW effects), for $t_{y_{2}}<t_{y}<t_{y_{1}}$; and (iii) a "spin frustrated" (SF, presumably metallic) phase, for $t_{y}>t_{y_{2}}$. We expect distinct boundaries between these regions.

To provide partial quantitative support for our qualitative considerations, we study the $(x-x$ component of the) bond order and the spin-density "structure factors",

$$
\chi_{B O W}(\bar{q})=\frac{1}{N} \sum_{i, j} e^{i \bar{q} \cdot\left(\vec{r}_{i}-\vec{r}_{i}\right)}\left(\left\langle B_{i, i+x} B_{j, j+x}\right\rangle-\left\langle B_{i, i+x}\right\rangle\left\langle B_{j, j+x}\right\rangle\right),
$$

where $B_{i, i+x}=\sum_{\sigma}\left(c_{i, \sigma}^{\dagger} c_{i+x, \sigma}+h . c.\right)$, and

$$
\chi_{S D W}(\vec{q})=\frac{1}{N} \sum_{i, j} e^{i \vec{q} \cdot\left(\vec{r}_{i}-\vec{r}_{j}\right)}\left\langle\left[n_{i+1, \uparrow}-n_{i+l, 1}\right]\left[n_{i, \uparrow}-n_{i, \downarrow}\right]\right\rangle,
$$

as functions of anisotropy. In the 1-D limit, we carried out quantum Monte Carlo (QMC) simulations for a chain of 128 atoms at temperature $\mathrm{T}=1 / 32$ in units of $t_{x}$; for $t_{x}=0.2 \mathrm{eV}$, 'is corresponds to $70^{\circ} \mathrm{K}$, higher than temperatures of interest in FISDW but reasonably close to ty $\mathrm{L}^{\prime}$ ical organic SP 


\begin{tabular}{|c|ccc|cc|cccc|}
\hline & \multicolumn{2}{|c|}{$\chi_{B O W}\left(q_{x}\right) U=3, V_{x}=1$} & \multicolumn{2}{c|}{$\chi S D W\left(q_{x}\right)$} & $U=3, V_{x}=1$ & \multicolumn{2}{|c|}{$\chi S D W\left(q_{x}\right) U=0, V_{x}=0$} \\
$t_{y}$ & $q_{x}=0$ & $q_{x}=\pi / 2$ & $q_{x}=\pi$ & $q_{x}=0$ & $q_{x}=\pi / 2$ & $q_{x}=\pi$ & $q_{x}=0$ & $q_{x}=\pi / 2$ & $q_{x}=\pi$ \\
\hline 0.0 & 0.2050 & 0.7078 & 0.2163 & 0.0008 & 0.6643 & 0.4721 & 0.1163 & 0.4957 & 0.5000 \\
0.5 & 0.2699 & 0.6981 & 0.2829 & 0.0222 & 0.7422 & 0.4582 & 0.3010 & 0.4750 & 0.5000 \\
0.8 & 0.6146 & 0.9474 & 0.6146 & 0.2696 & 0.6307 & 0.4968 & 0.4000 & 0.4825 & 0.5000 \\
1.0 & 0.9041 & 1.0066 & 0.8073 & 0.3099 & 0.5638 & 0.4941 & 0.4418 & 0.5000 & 0.5000 \\
\hline
\end{tabular}

Table 1: Structure factors versus $q_{x}$ for various values of $t_{y}$. Columns 2-4 are $\chi_{B O W}$ for $U=3, V_{x}=1$; columns 5-7 are $\chi_{S L W}$ f: $U=3, V_{x}=1$; columns 8-10 are $\chi_{S D W}$ for $U=0, V_{x}=0$; all units $t_{x}=1$.
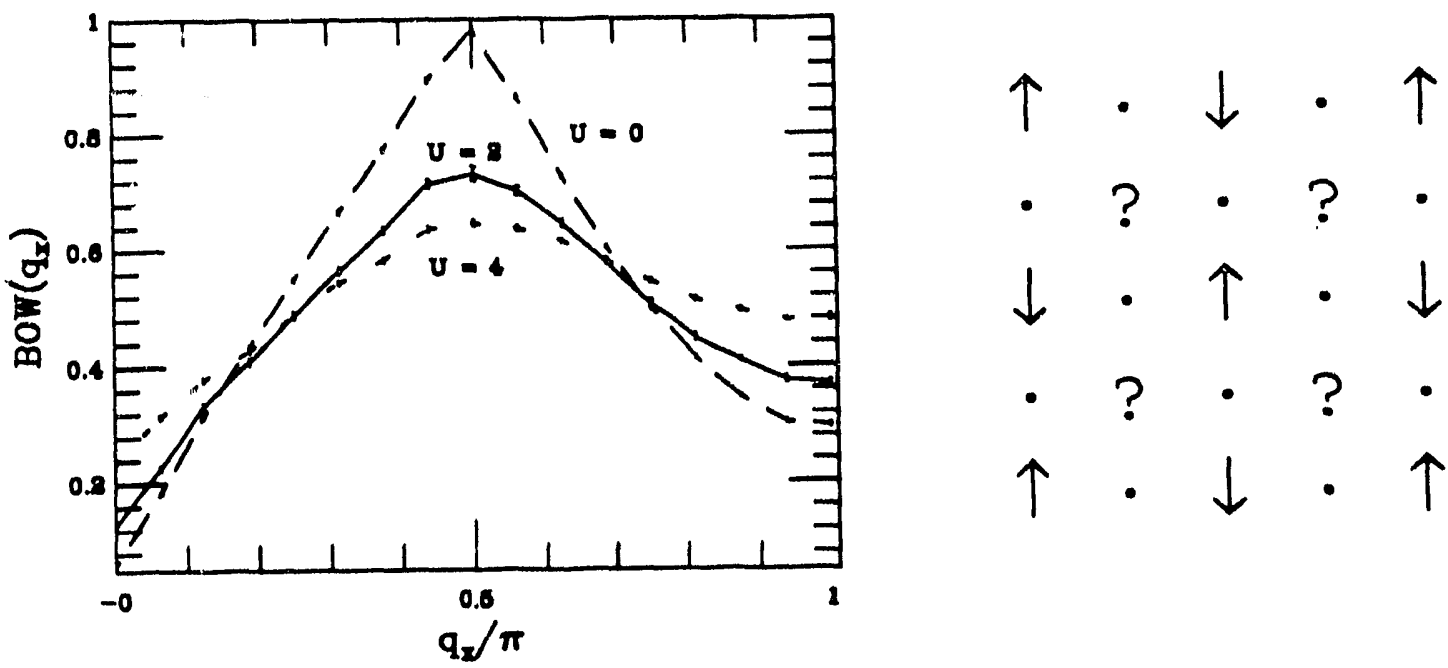

Fig. 1 (left). A small section of a 2-D lattice showing a spin configuration at $1 / 4$-filling; note that the antiferromagnetic couplings in the $\vec{x}, \vec{y}$ and $\vec{x}+\vec{y}$ directions lead to frustration on the sites labeled '?'.

Fig. 2 (right). $\chi_{B O W}$ for the 1-D Hubbard Model from a QMC calculation with $\mathrm{N}=128, T=t_{x} / 32$, and $U / t_{x}=0,2$, and 4 .

temperatures. As seen in Fig. 2, the $\chi_{B O W}(q)$ peaks at $2 k_{F}$ for values of $U$ in the weak to intermediate coupling range. Although (unlike the case for $\rho=1$ ) for $\rho=0.5$ the Coulomb interaction suppresses the BOW monotonically, a logarithmic divergence always exists [11], suggesting that a $2 \mathrm{k}_{F}$ BOW instability will occur for any finite electron-lattice coupling. For 2D lattices, except in the case $\rho=1$, similar QMC simulations are not possible because of the well-known "negative sign" problem. We have therefore done exact calculations for a $4 \times 4$ rectangular lattice. Finite size scaling is not possible currently; thus for comparison we use the $U=V_{x}=V_{y}=0$ case, for which analytical results are available. For hrevity we present results only for $U=3$ and $V_{x}=1$ (in units of $t_{x}=1$ ) and always with $V_{y} / V_{x}=t_{y} / t_{x}$. Finally, we present here results for $q_{y}=\pi$ only, as correlation functions along $\left(q_{x}, \pi\right)$ direction are the most relevant for describing SP or SDW transitions in 2D. [10] As seen in Table 1, the $q_{x}=\pi / 2$ ) BOW is gradually weakened as $t_{y}$ increases. In an infinite system we expect the reduction in the amplitude of the BOW with increasing $t_{y}$ to be faster. As Table 1 also shows, the amplitude of the $q_{x}=\pi / 2$ ) SDW, enhanced by the Coulomb interactions, first increases with $t_{y}$, but then peaks and actually decreases, indicating the frustration discussed above and sketched in Fig. 1.

\section{COMPARISON TO EXPERIMENT AND DISCUSSION}

Clearly our 2-D results are on systems of such small size that no quantitative comparison to experiment is fruitful; for instance, in our calculations the SDW is enhanced until a fairly large value of $t_{y}$ is reached, only beyond which the SDW amplitude begins to decrease. We focus thus on the qualitative 
the behavior of different classes of $2: 1$ materials.

(i) TMTTF salts. These are known to be the most 1-D in character. With some exceptions ((TMTTF $)_{2} \mathrm{Br}$, for example) a low temperature SP phase is the norm. Under pressure, the SP phase gives way to a SDW in (TMTTF) ${ }_{2} \mathrm{PF}_{B}$, with $T_{S D W}$ initially increasing with pressure and then exhibiting a maximum and derreasing [12]. Our approach explains this, since pressure increases $t_{y}$, first taking the system from the $t_{y}<t_{y_{1}}$ region to the $t_{y_{1}}<t_{y}<t_{y_{3}}$ region and then finally into the spin frustration regime.

(ii) TMTSF salts. These are the materials in which FISDW and VFHT are seen. The effect of the magnetic field is opposite to that of pressure; $\mathrm{H} \neq 0$ makes the system more one dimensional and takes it from the frustrated $t_{y}>t_{y_{2}}$ region to the $t_{y_{1}}<t_{y}<t_{y_{2}}$ region. However, in the VHFT region, transition to the $t_{y}<t_{y_{1}}$ "phase" is expected; thus we predict a SP phase, separated by a distinct boundary, from the "high" temperature phase at large H.[13] The SP phase will have all the characteristics of a semiconductor, including diamagnetism. Also, within our model FISDW should not necessarily be limited to superconducting TMTSF salts [8], although the experimental conditions under which the FISDW might appear are not clear, since application of both pressure and magnetic field may be necessary' to be in the right regime.

(iii) BEDT-TTF and MDT-TTF salts. These materials are the most strongly 2-D and are thus likely in the $t_{v}>t_{y_{2}}$ region where no SDW is expected. However, the crystal structures of these materials can vary from salt to salt, and sometimes several modifications are known even with the same anion. This is especially true for the MDT-TTF salts with asymmetric cations. Nakamura et al. have recently shown that (MDT-TTF $)_{2} \mathrm{Au}(\mathrm{CN})_{2}$ exhibits a SDW below $20^{\circ} \mathrm{K}$, but the SDW state is easily suppressed by application of pressure [14]. Although this is opposite to the effect seen in (TMTTF) ${ }_{2} \mathrm{PF}_{6}$, within

our approach it can be explained by assuming that at ambient pressure (MDT-TTF) ${ }_{2} \mathrm{Au}(\mathrm{CN})_{2}$ is in the $t_{y_{1}}<t_{y}<t_{y_{2}}$ region, so that pressure can take it into the SF regime.

We are grateful to Victor Yakovenko and Paul Chaikin for valuable discussions at the ICSM '90.

\section{REFERENCES}

1. X. Yan et al, in D. Jerome and L. Caron (eds.), Low Dimensional Conductors and Superconductors, Plenum, New York, 1987, 211.

2. L. J. Azevedo et al., Phys. Rev. Lett. 48 (1982) 826.

3. L.P. Gorkov and A.G. Lebed, J. de Phys. Lett., 43 (1984) L433.

4. G. Montasobaux, M. Heritier, and P. Lederer, Phys. Rev. Lett. 55 (1985) 2078.

5. K. Yamä̈, J. Phys. Soc. Jpn. 58 (1987) 1101.

6. T. Osada et al., Sol. St. Commun. 60 (1986) 441.

7. R.V. Chamberlin et al., Phys. Rev. Lett. 60 (1988) 1189.

8. V.M. Yakovenko, Sov. Phys. JE'TP, 66 (1987) 355.

9. S. Mazumdar and A.N. Bloch, Phys. Rev. Lett. 50 (1983) 207.

10. S. Mazumdar, Phys. Rev. B36 (1988) 9546.

11. J. E. Hirsch and D. J. Scalapino, Phys. Rev. B29, 5554 (1984).

12. F. Creuzet et al., Syn. Metals, 19 (1987) 289 and references therein.

13. See P. Chaikin et al., these Proceedings, for possible confirmation of this expectation.

14. T. Nakamura et al., Sol. St. Commun. 75 (1990) 583. 

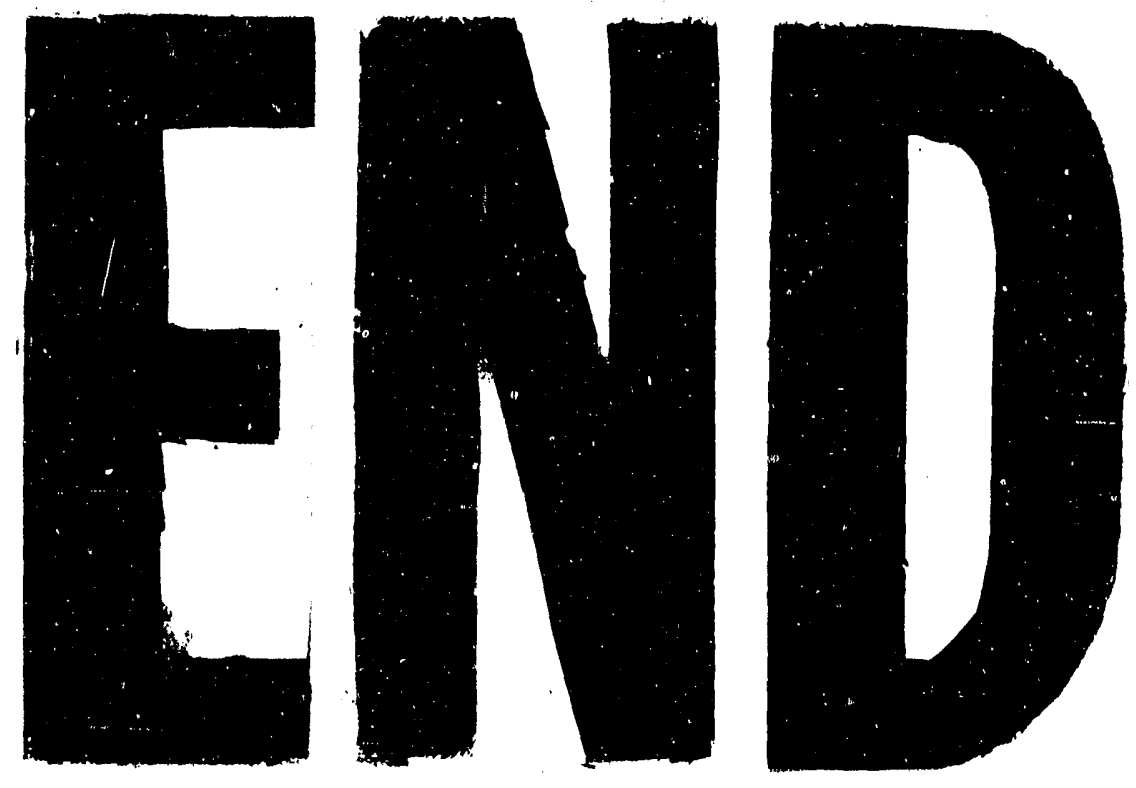

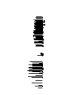

:
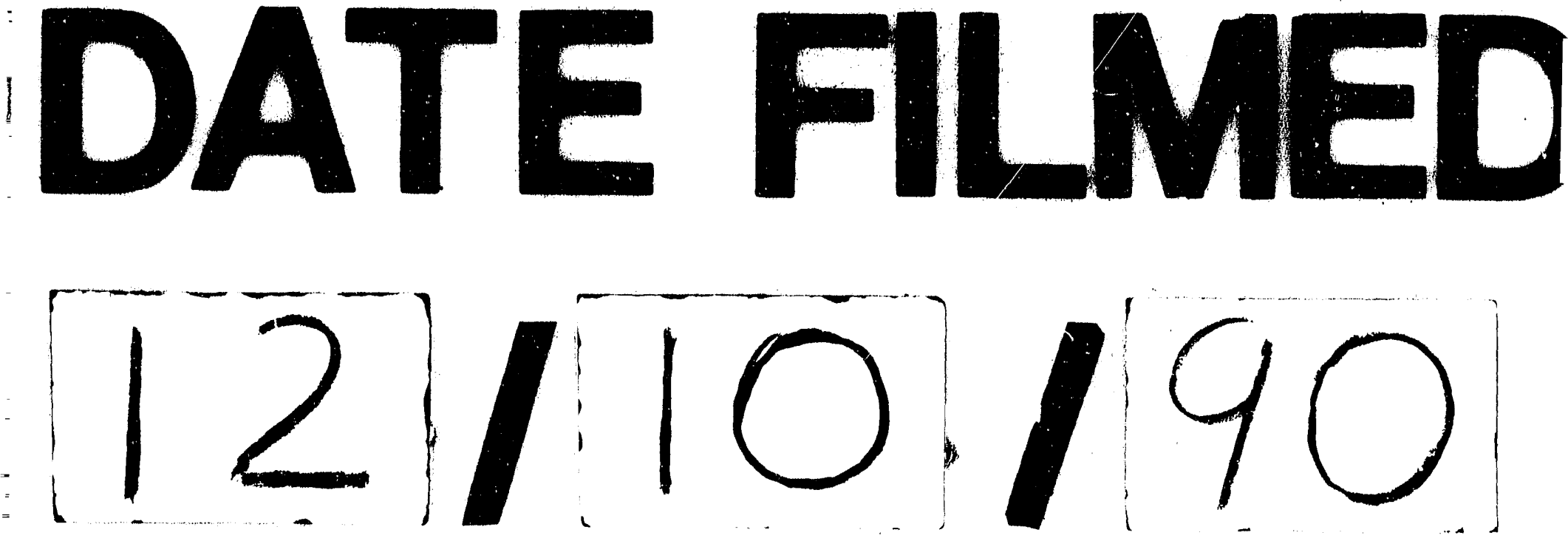
